\title{
Medical-Grade Honey as an Alternative Treatment for Antibiotics in Non-Healing Wounds-A Prospective Case Series
}

\author{
Adéla Holubová $^{1,2, *}$, Lucie Chlupáčová ${ }^{2}$, Lada Cetlová ${ }^{3}$, Niels A. J. Cremers ${ }^{4}\left(\mathbb{D}\right.$ and Andrea Pokorná ${ }^{3,5}$ (D) \\ 1 Faculty of Health and Social Sciences, University of Bohemia, 37011 České Budějovice, Czech Republic \\ 2 DiaPodi Care spol. s r.o., 39201 Soběslav, Czech Republic; diapodicare@seznam.cz \\ 3 Department of Health Sciences, College of Polytechnics Jihlava, 58601 Jihlava, Czech Republic; \\ lada.cetlova@vspj.cz (L.C.); apokorna@med.muni.cz (A.P.) \\ 4 Triticum Exploitatie BV, 6222 NK Maastricht, The Netherlands; niels@mesitran.com \\ 5 Department of Nursing and Midwifery, Faculty of Medicine, Masaryk University, \\ 62500 Brno, Czech Republic \\ * Correspondence: adela.holubova@diapodicare.cz; Tel.: +420-774-672-220
}

Citation: Holubová, A.; Chlupáčová, L.; Cetlová, L.; Cremers, N.A.J.;

Pokorná, A. Medical-Grade Honey as an Alternative Treatment for Antibiotics in Non-Healing Wounds-A Prospective Case Series. Antibiotics 2021, 10, 918. https:// doi.org/10.3390/antibiotics10080918

Academic Editor: Piotr Szweda

Received: 25 June 2021

Accepted: 25 July 2021

Published: 28 July 2021

Publisher's Note: MDPI stays neutral with regard to jurisdictional claims in published maps and institutional affiliations.

Copyright: (c) 2021 by the authors. Licensee MDPI, Basel, Switzerland. This article is an open access article distributed under the terms and conditions of the Creative Commons Attribution (CC BY) license (https:// creativecommons.org/licenses/by/ $4.0 /)$.

\begin{abstract}
Non-healing wounds are usually colonised by various types of bacteria. An alternative to antibiotic treatment in patients with infected wounds with local signs of inflammation may be medical-grade honey (MGH), which favourably affects the healing process with its antimicrobial, antioxidant, anti-inflammatory, and immunomodulatory properties. The objective of this study was to evaluate the effect of MGH therapy on the healing process of non-healing wounds of various aetiologies and different wound colonisations. Prospective, observation-intervention case studies $(n=9)$ of patients with wounds of various aetiologies (venous leg ulcers, diabetic foot ulcers, surgical wound dehiscence) are presented. All wounds were treated with MGH and the healing trajectory was rigorously and objectively monitored. In all cases, pain, odour, and exudation were quickly resolved, which led to an improvement in the quality of life of patients. Despite the proven bacterial microflora in wounds, antibiotic treatment was not necessary. The effects of MGH alleviated the signs of local infection until their complete elimination. In eight out of nine cases, the non-healing wound was completely healed. MGH has antimicrobial, anti-inflammatory, and antioxidant effects in wounds of various aetiologies and forms an effective alternative for the use of antibiotics for treating locally infected wounds.
\end{abstract}

Keywords: medical grade honey; antibiotic replacement; infections; wounds; objective wound assessment

\section{Introduction}

The Wound Healing Society has defined four categories of non-healing ulcers based on their aetiology: pressure ulcers, diabetic ulcers, and ulcers due to venous insufficiency or arterial insufficiency [1]. A non-healing wound, also called a hard to heal or chronic wound, is damage to the skin that heals unusually slow. Depending on the definition, a non-healing wound is usually present for at least 4 weeks [2]. Non-healing wounds have a major impact on the patient's quality of life $[3,4]$. Therefore, the main goal in the treatment of non-healing wounds is not only their healing but also the alleviation of unpleasant wound symptoms, such as pain and malodour [5]. An objective assessment of non-healing wounds, including the overall condition of the patient, is very important to develop a realistic treatment plan and to determine cost-effectivity [6-9].

The wound healing process is complex and dynamic [10,11]. Non-healing wounds are often critically colonized by various types of microorganisms. Local signs of inflammation in the wound include redness, oedema, increase in local temperature, tissue damage, and pain. In difficult-to-heal or non-healing wounds, they also include prolonged healing, the presence of abnormal granulation tissue, increase in wound size, and increased amount 
of exudate [12]. Antibiotic treatment based only on the presence of bacteria is not a good practice and is potentially dangerous with respect to the formation of polyresistant microbial strains, and ultimately leads to increased therapy costs and resource wastage $[13,14]$. The amount and behaviour of bacteria in wounds varies from contamination to invasive infection. Biofilm is often present, which is a bacterial colony surrounded by an extracellular matrix consisting of polysaccharides forming a shield to antimicrobial agents and increasing resistance [15]. One of the options to reduce the bioburden of the wound bed is sharp debridement. Debridement refers to a process in which avital tissue, coatings, microbial load (including biofilms), and tissue debris are removed from a wound [16-18]. Sharp debridement accelerates wound cleansing and shortens the total time required for tissue repair, but has low selectivity and often damages vital tissues $[19,20]$. Therefore, it is appropriate to use less invasive but similarly effective autolytic debridement techniques. One of them is the use of medical-grade honey (MGH).

Honey has been used to treat wounds and local infections for more than 5000 years [21]. MGH is carefully selected and gamma-sterilized in order to ensure safe use for wound care $[22,23]$. MGH has a positive effect on the healing process with its antimicrobial, antioxidant, anti-inflammatory, and immunomodulatory properties. It also stimulates the production of hydrogen peroxide at a concentration that is not toxic to damaged tissues [24,25]. It also supports the activity of the immune system, debridement, and stimulates regenerative processes in the wound [26]. MGH decreases wound healing time and is cost-effective [27]. The aim of our study was to assess the effect of MGH on the healing of non-healing wounds. In this prospective case series, nine patients with infected non-healing wounds were treated with MGH in the absence of antibiotics. Our hypothesis was that MGH could replace the use of antibiotics in locally infected wounds and promote debridement, wound healing, and patients' quality of life.

\section{Results}

\subsection{Case 1: Dehisced Surgical Wound}

A 57-year-old female patient presented with a dehisced surgical wound at her left breast following a breast-conserving operation as a result of being diagnosed with breast cancer (Figure 1a). Relevant comorbidities included ovarian cancer and having diabetes mellitus (DM) type 2, which could subsequently affect the healing. Previous treatments with sterile bioceramic dressings for three months were ineffective. The wound dimensions upon presentation were $6 \mathrm{~cm}$ in length, $1.5 \mathrm{~cm}$ in width, and ranging from $0.5 \mathrm{~cm}$ to $2 \mathrm{~cm}$ in depth (deeper towards axilla). The wound consisted mainly (roughly $95 \%$ ) of granulation tissue and $5 \%$ slough. Low levels of exudate (thin, water-like) were produced. Local signs of infection included delayed healing, pain, and redness. The pain was scored on a visual analogue (VAS)-scale by the patient for pain level during the daytime and during wound care treatment. Pain level was 5 during the daytime and 8 during treatment. L-Mesitran ${ }^{\circledR}$ Soft gel (MGH) was applied inside the lesion and followed by L-Mesitran ${ }^{\circledR}$ Tulle (MGH) to ensure contact to the wound. Mepilex foam (foam dressing) was applied as a secondary dressing. Wound dressings were performed by the patient at home at $72 \mathrm{~h}$ intervals for the first two weeks. Pain and redness disappeared after 14 days of treatment. Due to the positive evolution of the healing, the dressing changes interval were extended to every four days. The wound was completely healed after 35 days of MGH treatment without complications (Figure 1b). 


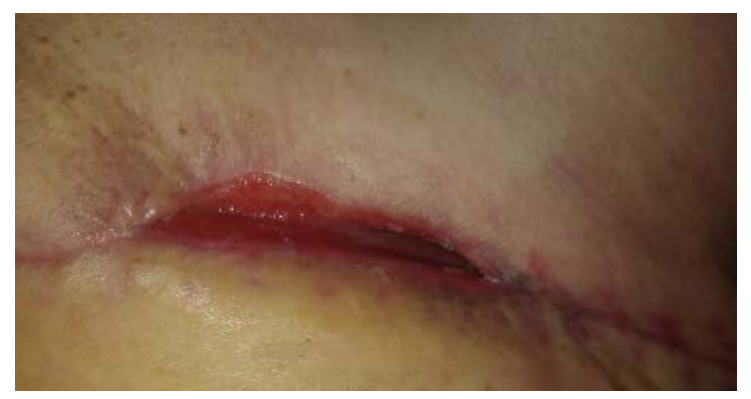

(a)

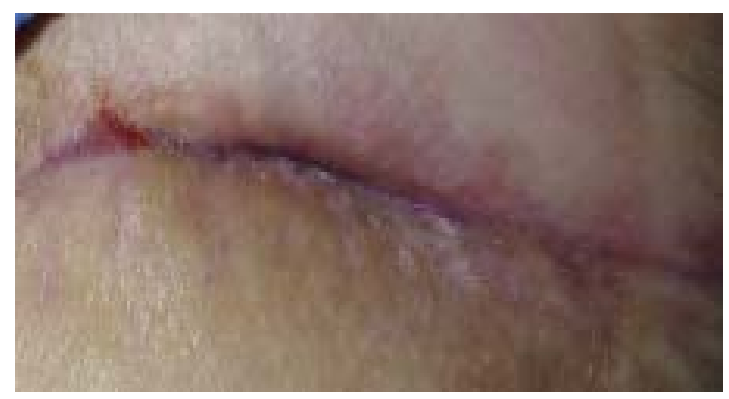

(b)

Figure 1. Case 1: Dehiscence of the surgical wound on the left breast. (a) Local finding at initial examination, day 0 (start of MGH treatment). (b) Complete wound healing on follow-up examination at day 35.

\subsection{Case 2: Venous Leg Ulcer}

A 43-year-old male patient presented with a venous leg ulcer at his right lower leg (Figure 2a). Relevant comorbidities included chronic venous insufficiency (CHVI), diabetes mellitus (DM), obesity (BMI 30), and repeated venous lower ulcers. Previous treatments with iodinated povidone solution for two months were ineffective. The wound dimensions upon presentation were $6 \mathrm{~cm}$ in length, $5 \mathrm{~cm}$ in width, and $1 \mathrm{~cm}$ in depth. The wound consisted of $5 \%$ of granulation tissue and $95 \%$ slough. Low levels of exudate (thin, water-like) were produced. Local signs of infection included pain, erythema, local warmth, exudate, delayed healing, and malodour. A microbiological swab was performed, in which Enterococcus faecalis (resistant to Trimethoprim + sulphonamide, neomycin, clindamycin, gentamicin; and sensitive to ampicillin, nitrofurantoin, norfloxacin, bacitracin, ciprofloxacin, and chloramphenicol) and Escherichia coli without resistance were detected (sensitive to ampicillin, aminopenicillin, cefuroxime, trimethoprim + sulphonamide, cefpodoxime, neomycin, gentamicin, ciprofloxacin, and chloramphenicol). Pain level was 8 during the daytime and 9 during treatment. L-Mesitran ${ }^{\circledR}$ Ointment (MGH) was applied to the wound (Figure 2b) and followed by L-Mesitran ${ }^{\circledR}$ Tulle (Figure 2c) to ensure contact to the wound bed. Suprasorb P foam (foam dressing) was applied as a secondary dressing. Wound dressings were performed by the patient at home at $48 \mathrm{~h}$ intervals for the first two weeks. After 20 days, the wound dimensions upon presentation were $4 \mathrm{~cm}$ in length, $4 \mathrm{~cm}$ in width, and $0.5 \mathrm{~cm}$ in depth (Figure $2 \mathrm{~d}$ ). The wound consisted of $20 \%$ of granulation tissue, 30\% epithelializing, and 50\% slough, and malodour and infection disappeared. Pain levels gradually decreased and after 20 days of treatment, the pain level was 2 (VAS). Due to the positive development of healing, the dressing changes were extended to every three days. The wound was completely healed after 67 days of MGH treatment without complications (Figure 2e).

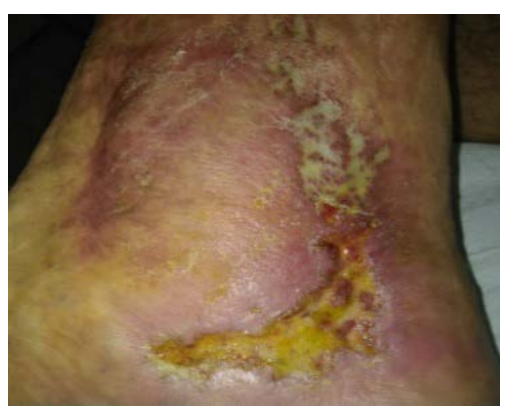

(a)

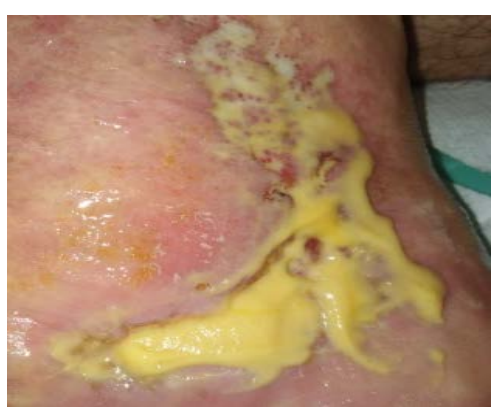

(b)

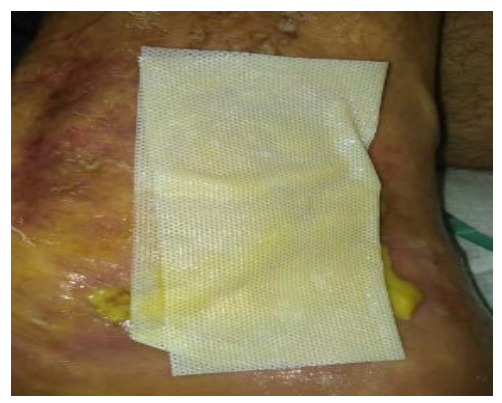

(c)

Figure 2. Cont. 


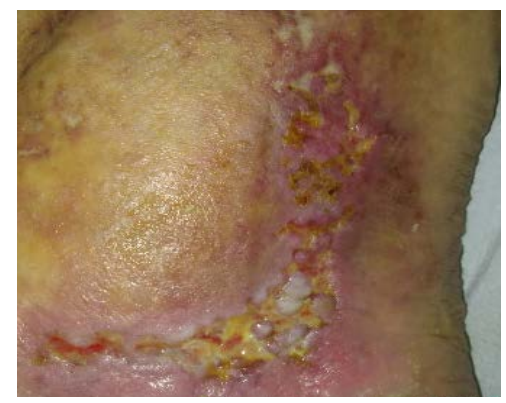

(d)

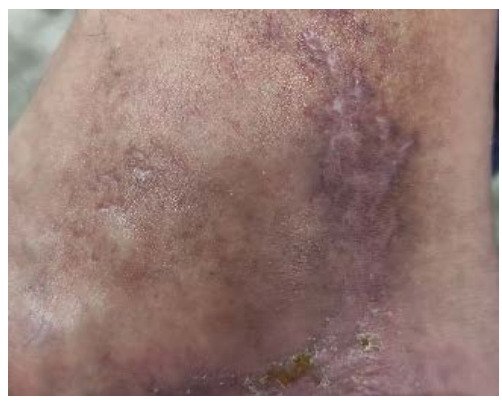

(e)

Figure 2. Case 2: Venous leg ulcer on the right lower leg. (a) Local finding at initial examination, day 0 (start of MGH treatment). (b) Example of L-Mesitran ${ }^{\circledR}$ Ointment application. (c) Example of L-Mesitran ${ }^{\circledR}$ Tulle application. (d) Follow-up examination at day 20. (e) Complete wound healing on follow-up examination at day 67.

\subsection{Case 3: Venous Leg Ulcer}

A 72-year-old male patient presented with a venous leg ulcer on his right lower leg (Figure 3a). Relevant comorbidities included CHVI, DM, hypertension (HT), and a medical history of thrombosis of the right lower leg without acute symptomatology. Previous treatments with iodinated povidone solution for six weeks were ineffective. Upon presentation, the wound dimensions were $14 \mathrm{~cm}$ in length, $4 \mathrm{~cm}$ in width, and $1 \mathrm{~cm}$ in depth. The wound consisted of $30 \%$ of granulation tissue and $70 \%$ slough. Medium levels of exudate (thin, water-like) were produced. Local signs of infection included pain, delayed healing, and malodour. A swab was performed in which Enterococcus faecalis (resistant to trimethoprim + sulphonamide, neomycin, clindamycin, and gentamicin; and sensitive to ampicillin, nitrofurantoin, bacitracin, ciprofloxacin, chloramphenicol) and Escherichia coli without resistance were detected (and sensitive to ampicillin, aminopenicillin, cefuroxime, trimethoprim + sulphonamide, cefpodoxime, gentamicin, ciprofloxacin, and chloramphenicol). Pain level was 6 during the daytime and 9 during treatment. L-Mesitran ${ }^{\circledR}$ Ointment was applied to the wound and followed by L-Mesitran ${ }^{\circledR}$ Tulle. Resposorb ${ }^{\circledR}$ Super (super absorbent dressing) was applied as a secondary dressing. Wound dressings were performed by the patient at home at $48 \mathrm{~h}$ intervals for the first two weeks. Pain levels gradually decreased and after 14 days of treatment, the pain was tolerated at pain level 1 (daytime) and 2 (during treatment-procedural pain). Due to the positive development of healing, the dressing changes were extended to every three days. After 42 days, only L-Mesitran ${ }^{\circledR}$ Tulle was applied to the wound. Suprasorb P foam was applied as a secondary dressing. The wound was completely healed after 79 days of MGH treatment without complications (Figure 3b).

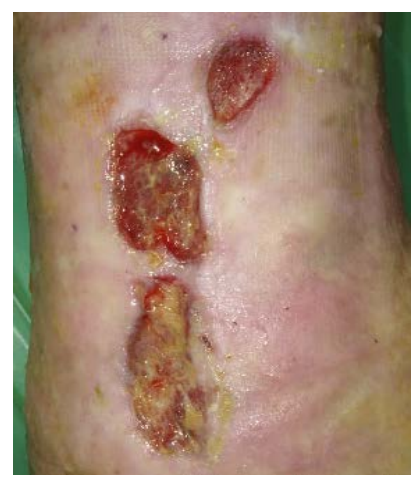

(a)

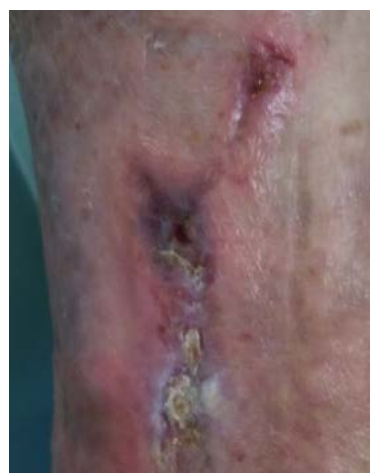

(b)

Figure 3. Case 3: Venous leg ulcer on the right lower leg. (a) Local finding at initial examination, day 0 (start of MGH treatment). (b) Complete wound healing on follow-up examination at day 79. 


\subsection{Case 4: Venous Leg Ulcer}

A 75-year-old female patient presented with a venous leg ulcer on her left lower leg (Figure 4a). Relevant comorbidities included CHVI, DM, HT, and the patient underwent varices surgery on the left lower leg in the past with no actual symptomatology. Previous treatments with antiseptic dressing with silver nanoparticles for two months were ineffective. Upon presentation, the wound dimensions were $1.5 \mathrm{~cm}$ in length, $1.5 \mathrm{~cm}$ in width, and $0.8 \mathrm{~cm}$ in depth (top wound) and $3 \mathrm{~cm}$ in length, $2 \mathrm{~cm}$ in width, and $0.8 \mathrm{~cm}$ in depth (lower wound), both in craniocaudal direction. The wound bed consisted of 30\% of granulation tissue and 70\% slough. Medium levels of exudate (thin, water-like) were produced. Local signs of infection included pain and delayed healing. A wound swab confirmed the presence of Staphylococcus aureus resistant to clindamycin and sensitive to trimethoprim + sulphonamide, norfloxacin, neomycin, bacitracin, chloramphenicol, and gentamicin. Pain level was 5 during the daytime and 7 during treatment (procedural pain). L-Mesitran ${ }^{\circledR}$ Ointment was applied to the wound and followed by L-Mesitran ${ }^{\circledR}$ Tulle. Resposorb ${ }^{\circledR}$ Super (foam dressing) was applied as a secondary dressing. Wound dressings were performed by the patient at home at $48 \mathrm{~h}$ intervals for the first two weeks. After 51 days, the wound dimensions were $6 \mathrm{~cm}$ in length, $6 \mathrm{~cm}$ in width, and $0.5 \mathrm{~cm}$ in depth (Figure $4 \mathrm{~b}$ ). The wound bed consisted of $70 \%$ of granulation tissue and $30 \%$ epithelialization tissue. Pain levels gradually decreased and after 14 days of treatment, the pain level during the daytime was 1 and during treatment it was rated at level 2. After the proposed topical treatment, the wound bed was cleansed, the granulation and epithelialization phases took place, but the wound area increased despite getting more superficial. There was also a reduction in wound exudate and pain, and local signs of infection disappeared. Due to these aspects, the dressing was changed to L-Mesitran ${ }^{\circledR}$ Net (MGH) (Figure 4c) and PermaFoam ${ }^{\circledR}$ classic (foam dressing) was used as a secondary dressing. Due to the positive development of healing, the dressing changes were extended to every three days. The wound was completely healed after 62 days of MGH treatment without complications (Figure 4d).

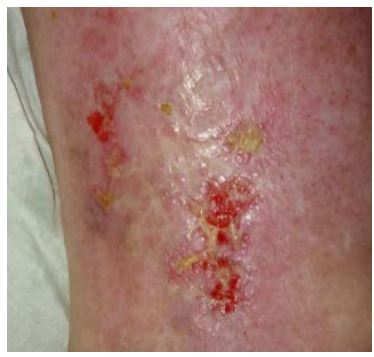

(a)

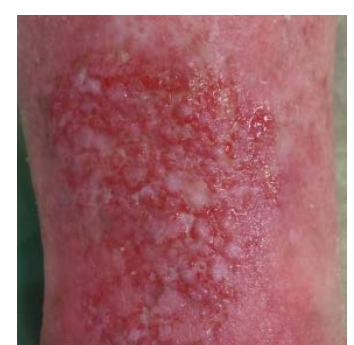

(b)

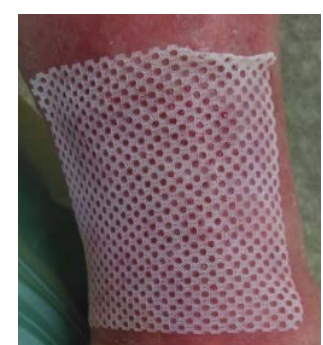

(c)

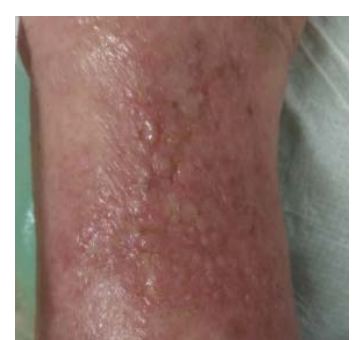

(d)

Figure 4. Case 4: Venous leg ulcer on the left lower leg. (a) Local finding at initial examination, day 0 (start of MGH treatment). (b) Follow-up examination at day 51. (c) Example of L-Mesitran ${ }^{\circledR}$ Net application. (d) Complete wound healing on follow-up examination at day 62.

\subsection{Case 5: Diabetic Foot Ulcer}

A 59-year-old male patient presented with a diabetic foot ulcer at his right foot (Figure 5a). Relevant comorbidities included repeated diabetic gangrene, repeated amputation of toes on the right foot, diabetic neuropathy, DM, HT, and obesity (BMI 32). Previous treatments with iodinated povidone solution for six weeks were ineffective. Upon presentation, the wound dimensions were $8 \mathrm{~cm}$ in length, $3 \mathrm{~cm}$ in width, and $5 \mathrm{~cm}$ in depth. The wound consisted of $80 \%$ of granulation tissue and $20 \%$ slough. High levels of exudate (thin, water-like) were produced. Local signs of infection included low level of neuropathic pain, exudate, delayed healing, and malodour. There was maceration, hyperkeratosis and callus in the peri-wound skin. A wound swab confirmed the presence of Proteus mirabilis (resistant to ampicillin, aminopenicillin, cefuroxime, trimethoprim + sulphonamide, cefpodoxime, gentamicin, ciprofloxacin, chloramphenicol, and cefotaxime; and sensitive to 
neomycin, amikacin, ceftazidime, ertapenem, meropenem), Staphylococcus aureus (resistant to clindamycin, gentamicin; and sensitive to cefoxitin, trimethoprim + sulphonamide, norfloxacin, neomycin, bacitracin, chloramphenicol, fusidic acid, and mupirocin) and Acinetobacter baumanii (resistant to ampicillin, aminopenicillin, cefuroxime, cefpodoxime, chloramphenicol, cefotaxime; and sensitive to trimethoprim + sulphonamide, neomycin, gentamicin, ciprofloxacin, amikacin, ceftazidime). Pain level was 1 during the daytime as well as during treatment (diabetic neuropathy). L-Mesitran ${ }^{\circledR}$ Ointment was applied to the wound, followed by L-Mesitran ${ }^{\circledR}$ Tulle (Figure 5 b) and Resposorb ${ }^{\circledR}$ Super as a secondary dressing. Wound dressings were performed by the patient at home at $48 \mathrm{~h}$ intervals due to bacterial findings and heavy wound exudation for first follow-up examination. On day 25, the wound dimensions were $5 \mathrm{~cm}$ in length, $1.5 \mathrm{~cm}$ in width, and $1 \mathrm{~cm}$ in depth (Figure 5c). The wound consisted of $90 \%$ of granulation tissue and $10 \%$ slough. Pain and odour levels gradually decreased over time and were completely absent on day 25 . After the proposed topical treatment, the wound area was reduced and the wound bed was cleaned. Due to the occurrence of maceration, likely due to non-compliance to the treatment, topical treatment was slightly adjusted. The application of L-Mesitran ${ }^{\circledR}$ Ointment was omitted and the patient was advised to continue treatment at home at $48 \mathrm{~h}$ intervals due to moderate wound exudation.

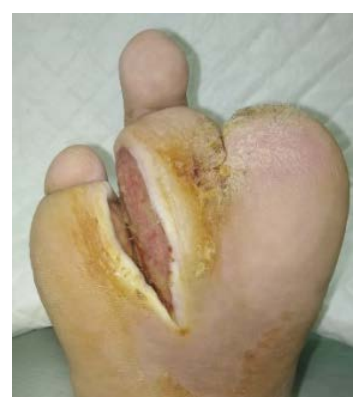

(a)

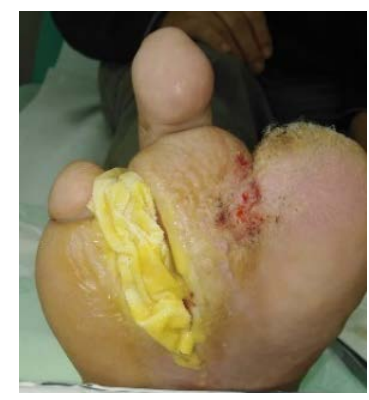

(b)

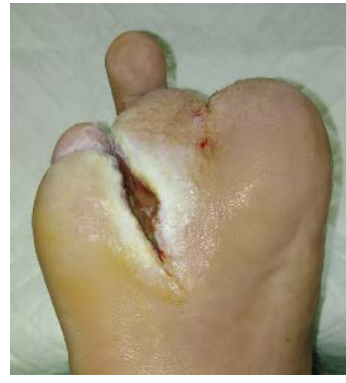

(c)

Figure 5. Case 5: Diabetic foot ulcer on the right foot. (a) Local finding at initial examination, day 0 (start of MGH treatment). (b) Example of L-Mesitran ${ }^{\circledR}$ Tulle application. (c) Improved wound healing on follow-up examination at day 25.

\subsection{Case 6: Venous Leg Ulcer}

A 54-year-old male patient presented with a venous leg ulcer at his right lower leg (Figure 6a). Relevant comorbidities included CHVI, HT, DM, hyperlipidaemia, and hyperuricemia. Previous treatments with iodinated povidone solution for seven weeks were ineffective. Upon presentation, the wound dimensions were $6 \mathrm{~cm}$ in length, $6 \mathrm{~cm}$ in width, and $0.5 \mathrm{~cm}$ in depth. The wound consisted of $95 \%$ of granulation tissue and $5 \%$ slough. Low levels of exudate (thin, water-like) were produced. Local signs of infection included pain and delayed healing. A wound swab confirmed the presence of Enterococcus faecalis (resistant to trimethoprim + sulphonamide, neomycin, clindamycin, gentamicin; and sensitive to ampicillin, nitrofurantoin, ciprofloxacin, and chloramphenicol). Pain level was 6 during the daytime and 8 during treatment. L-Mesitran ${ }^{\circledR}$ Foam was applied to the wound (Figure 6b). Wound dressings were performed by the patient at home at $72 \mathrm{~h}$ intervals. Pain levels gradually decreased over time and after 10 days of treatment, the pain level during daytime was 0 and 1 during treatment. The wound was completely healed after 15 days of MGH treatment without complications (Figure 6c). 


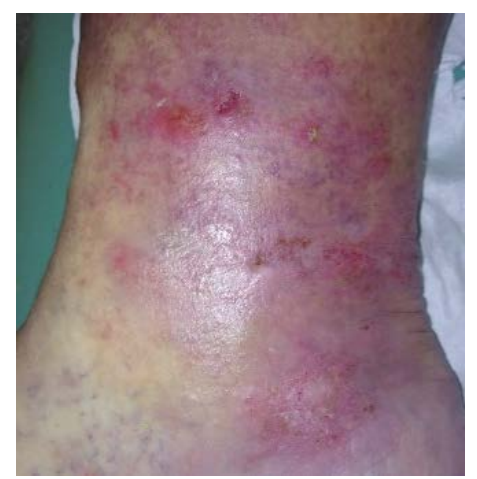

(a)

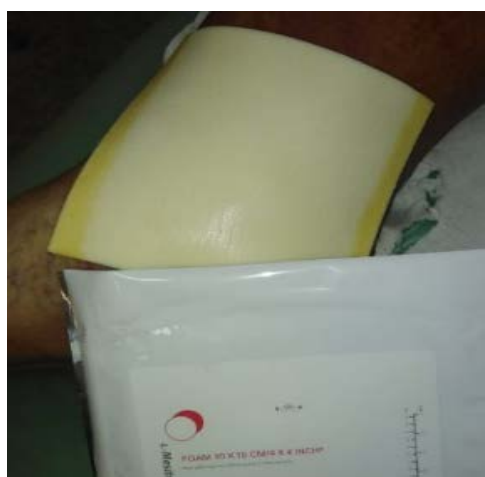

(b)

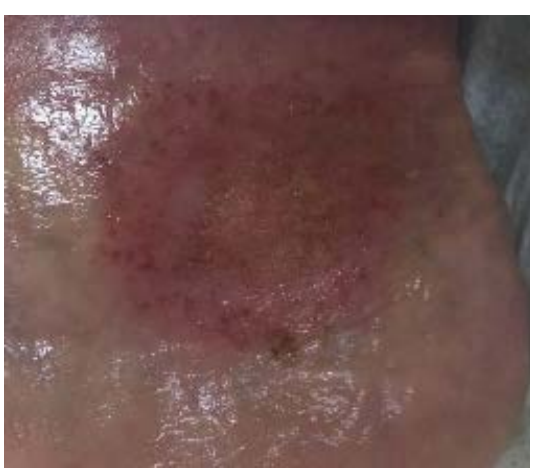

(c)

Figure 6. Case 6: Venous leg ulcer on the right lower leg. (a) Local finding at initial examination, day 0 (start of MGH treatment). (b) Example of L-Mesitran ${ }^{\circledR}$ Foam application. (c) Complete wound healing on follow-up examination at day 15.

\subsection{Case 7: Venous Leg Ulcer}

A 52-year-old male patient presented with bilateral venous leg ulcers (Figure 7a). Relevant comorbidities included CHVI, DM, HT hyperlipidaemia, hyperuricemia, morbid obesity (BMI 45), and repeated venous leg ulcers. Previous treatments with iodinated povidone solution for two months were ineffective. Upon presentation, the wound dimensions on the right leg were $2 \mathrm{~cm}$ in length, $2 \mathrm{~cm}$ in width, and $1 \mathrm{~cm}$ in depth. On the left leg, the wound dimensions were $10 \mathrm{~cm}$ in length, $12 \mathrm{~cm}$ in width, and $1 \mathrm{~cm}$ in depth. The wound bed consisted of $80 \%$ of granulation tissue and $20 \%$ slough. Medium levels of exudate (thin, water-like) were produced. Local signs of infection included pain and delayed healing. A wound swab confirmed the presence of Proteus mirabilis (resistant to ampicillin, aminopenicillin, cefuroxime, trimethoprim + sulphonamide, cefpodoxime, gentamicin, ciprofloxacin, and cefotaxime and sensitive to neomycin, amikacin, ceftazidime, meropenem). Staphylococcus aureus (resistant to clindamycin; and sensitive to trimethoprim + sulphonamide, norfloxacin, neomycin, bacitracin, and chloramphenicol). Pain level was 5 during daytime and 8 during treatment. L-Mesitran ${ }^{\circledR}$ Ointment was applied to the wound (Figure $7 \mathrm{~b}$ ) and followed by L-Mesitran ${ }^{\circledR}$ Tulle (Figure 7c) to ensure contact to the wound. Vacutex ${ }^{\circledR}$ (capillary action dressing) was applied as a secondary dressing (Figure $7 \mathrm{~d}$ ). Dressing changes were performed by the patient at home at $48 \mathrm{~h}$ intervals because of the heavy bacterial colonization and moderate wound exudation. Pain levels gradually decreased and after 14 days of treatment, the pain level was 1 during the daytime and 2 during treatment. After 14 days of treatment, the interval of dressing changes was prolonged to $72 \mathrm{~h}$. Since the wound became more superficial, L-Mesitran ${ }^{\circledR}$ Ointment was omitted from the treatment and L-Mesitran ${ }^{\circledR}$ Tulle was applied and PermaFoam ${ }^{\circledR}$ classic was used as a secondary dressing. The wound was completely healed after 54 days of MGH treatment without complications (Figure 7e).

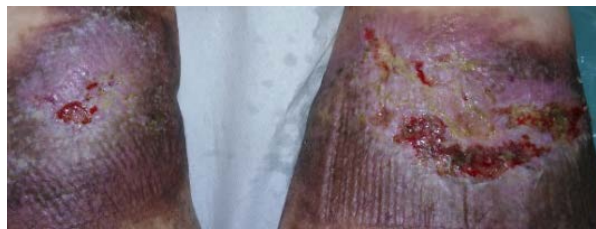

(a)

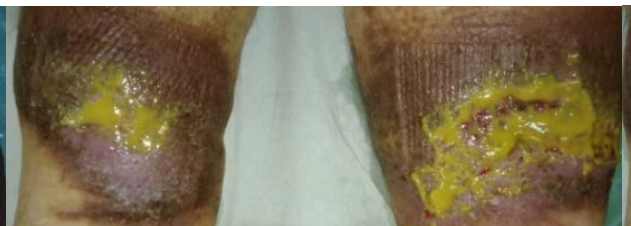

(b)

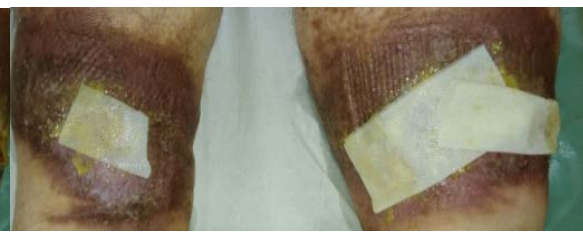

(c)

Figure 7. Cont. 


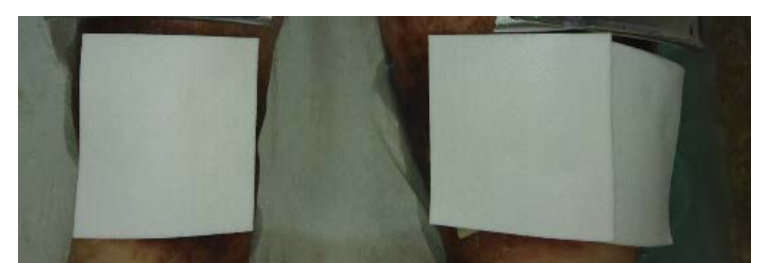

(d)

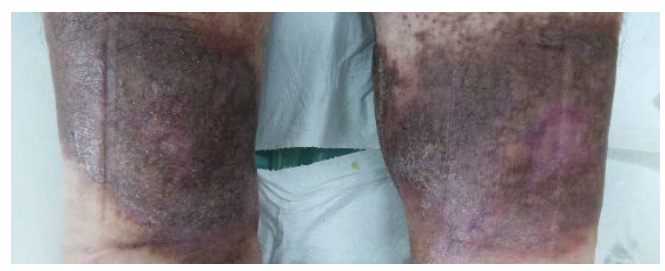

(e)

Figure 7. Case 7: Bilateral venous leg ulcers. (a) Local finding at initial examination, day 0 (start of MGH treatment). (b) Example of L-Mesitran ${ }^{\circledR}$ Ointment application. (c) Example of L-Mesitran ${ }^{\circledR}$ Tulle application. (d) Example of Vacutex ${ }^{\circledR}$ application. (e) Complete wound healing on follow-up examination at day 54.

\subsection{Case 8: Diabetic Foot Ulcer}

A 51-year-old female patient presented with a diabetic foot ulcer on her left foot (Figure 8a). Relevant comorbidities included DM, HT, morbid obesity (BMI 45), and repeated wounds on the right foot due to diabetic foot syndrome. Previous treatments with iodinated povidone solution for two months were ineffective. Upon presentation, the wound dimensions were $5 \mathrm{~cm}$ in length, $3 \mathrm{~cm}$ in width, and $1 \mathrm{~cm}$ in depth. The wound consisted of $90 \%$ of granulation tissue, $5 \%$ slough, and $5 \%$ epithelialization tissue. Low levels of exudate (thin, water-like) were produced. Local signs of infection included pain and delayed healing. A wound swab confirmed the presence of Proteus mirabilis (resistant to ampicillin, aminopenicillin, cefuroxime, trimethoprim + sulphonamide, cefpodoxime, gentamicin ciprofloxacin, chloramphenicol, cefotaxime; and sensitive to neomycin, amikacin, ceftazidime, ertapenem, and meropenem). Staphylococcus aureus (resistant to clindamycin, gentamicin; and sensitive to trimethoprim + sulphonamide, norfloxacin, neomycin, bacitracin, chloramphenicol, fusidic acid, mupirocin) and Enterococcus faecalis (resistant to trimethoprim + sulphonamide, neomycin, clindamycin, gentamicin; and sensitive to ampicillin, nitrofurantoin, norfloxacin, bacitracin, ciprofloxacin, and chloramphenicol). Pain level was 5 during the daytime and 8 during treatment. L-Mesitran ${ }^{\circledR}$ Ointment was applied to the wound and L-Mesitran ${ }^{\circledR}$ Foam was applied as a secondary dressing. Dressing changes were performed by the patient at home at $48 \mathrm{~h}$ intervals due to bacterial findings. On day 21, the wound dimensions upon presentation were $1.5 \mathrm{~cm}$ in length, $0.5 \mathrm{~cm}$ in width, and $0.5 \mathrm{~cm}$ in depth (Figure $8 \mathrm{~b}$ ). Pain levels gradually decreased and after 21 days of treatment, the pain level was 1 during the daytime and during treatment. The interval of the dressing changes was prolonged to $72 \mathrm{~h}$ and continued with applying only L-Mesitran ${ }^{\circledR}$ Foam. The wound was completely healed after 44 days of MGH treatment without complications (Figure 8c).

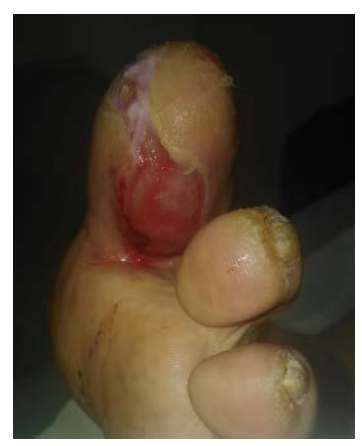

(a)

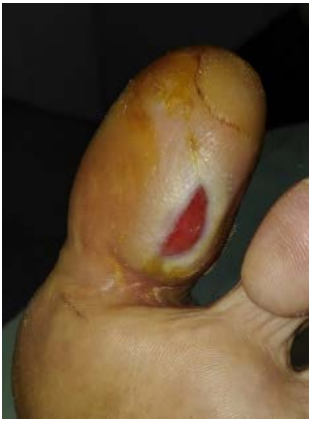

(b)

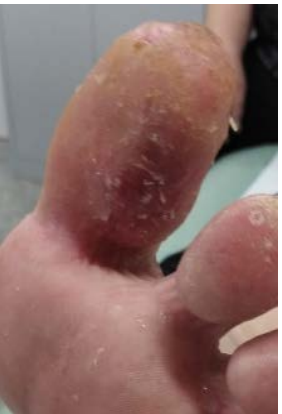

(c)

Figure 8. Case 8: Diabetic foot ulcer on the left foot. (a) Local finding at initial examination, day 0 (start of MGH treatment). (b) Follow-up examination at day 21. (c) Complete wound healing on follow-up examination at day 44. 


\subsection{Case 9: Venous Leg Ulcer}

A 49-year-old male patient presented with a venous leg ulcer at his right lower leg (Figure 9a). Relevant comorbidities included DM, HT, hyperlipidaemia, and a medical history of thrombosis of the right lower leg without acute signs of ischemia. Previous treatments with antiseptic dressing with silver nanoparticles for seven weeks were ineffective. Upon presentation, the wound dimensions were $5 \mathrm{~cm}$ in length, $3 \mathrm{~cm}$ in width, and $1.5 \mathrm{~cm}$ in depth. The wound consisted of $90 \%$ of granulation tissue, $5 \%$ slough, and $5 \%$ epithelialization tissue. Low levels of exudate (thin, water-like) were produced. Local signs of infection included pain, malodour, and delayed healing. A wound swab confirmed the presence of Enterococcus faecalis (resistant to trimethoprim + sulphonamide, neomycin, clindamycin, gentamicin; and sensitive to ampicillin, nitrofurantoin, bacitracin, ciprofloxacin, chloramphenicol). Pain level was 5 during the daytime and 8 during treatment. L-Mesitran ${ }^{\circledR}$ Soft (MGH) was applied to the wound and L-Mesitran ${ }^{\circledR}$ Foam (Figure 9b) was applied as a secondary dressing. Dressing changes were performed by the patient at home at $48 \mathrm{~h}$ intervals. Pain and malodour levels gradually decreased and after 14 days of treatment, the pain was absent during the daytime (VAS score of 0 ) and at level 1 during treatment. Only L-Mesitran ${ }^{\circledR}$ Foam was applied. The wound was completely healed after 17 days of MGH treatment without complications (Figure 9c).

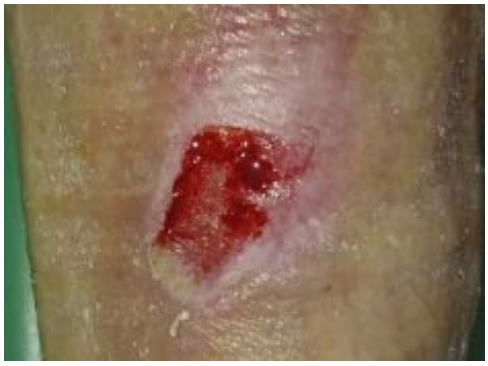

(a)

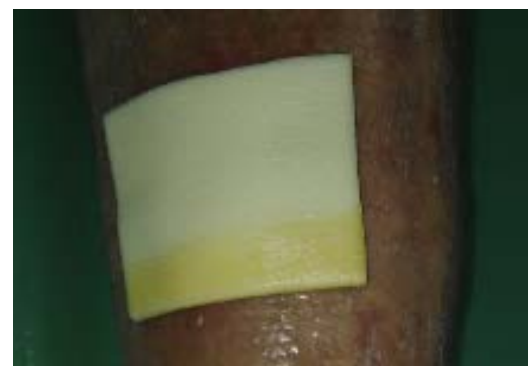

(b)

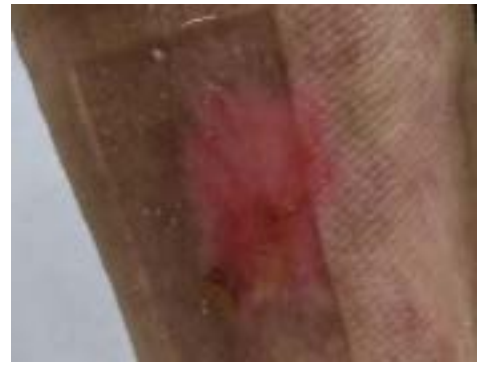

(c)

Figure 9. Case 9: Venous leg ulcer on the right lower leg. (a) Local finding at initial examination, day 0 (start of MGH treatment). (b) Example of L-Mesitran ${ }^{\circledR}$ Foam application. (c) Complete wound healing on follow-up examination at day 17.

\section{Discussion}

In all presented cases, the healing process was positively affected; the wound bed was cleansed; and the pain, odour, and exudation were completely eliminated. Despite the proven microbial burden in the wound by means of swab and cell culture examination, antibiotic treatment was not initiated and treated locally with only MGH. Signs of local infection were eliminated by the effect of MGH in the applied materials. In eight out of nine cases, the non-healing wound was completely healed. In one case (case 5) treatment with MGH continues. However, even in this case, there was a significant improvement in the local finding, but due to the low patient compliance, we have no further information. After proper training, the patients were able to perform the wound dressings themselves or with the help of family members at home, and they all considered it easy. No adverse effects of the applied topical material with MGH were observed.

The costs of wound management in developed countries are around 1-4\% of total health care expenditure $[15,28]$. The number of non-healing wounds is expected to increase, due to longevity and comorbidities, such as obesity and diabetes mellitus, and hence, elevate economic impact $[2,29,30]$. MGH enables a faster healing process and can resolve local infections without the need for antibiotic treatment, and thus forms a potent alternative treatment.

A total of nine patients with wounds of various aetiologies were included in the study. MGH promoted autolytic debridement, led to the cleansing of the wound bed, and induced granulation tissue formation and epithelialization. This is in line with previous studies $[14,31]$. Debridement and cleansing can be attributed to the moist wound 
environment, its acidification, the osmotic activity, and oxygenation of the wound environment $[14,31,32]$. The prevalence of biofilms in non-healing wounds is estimated to be approximately $60 \%[33,34]$. Biofilms have a high capacity for bacterial resistance and show increased resistance to host cellular responses and antiseptics [15,35]. MGH is effective in removing coating and necrosis from the wound bed, and thus may work in cases where antibiotics are ineffective [24]. Furthermore, MGH was also effective in eradicating MRSA in venous leg ulcers, so antibiotic-resistant strains can also be eliminated. The broad-spectrum antimicrobial activity of MGH on Staphylococcus aureus, Pseudomonas aeruginosa, and Streptococcus pathogens have been also confirmed by others [21,31,36]. Due to its antimicrobial mechanisms, including acidic $\mathrm{pH}$, osmotic activity, and slow release of hydrogen peroxide, MGH is effective against a wide range of pathogens, including multi-resistant bacteria, fungi, and viruses [13,37,38]. Moreover, the use of MGH in wound management can reduce the use of antibiotics and topical antiseptics [32]. Repeated use of MGH materials is without risk of developing resistance [13,21]. In our study, all patients had local signs of infection and microbial burden in the wound bed was proven in eight out of nine patients (wound swab was not taken in one case). Antibiotic treatment was required in none of the cases. We have verified that topical treatment with MGH is a safe and easy-to-use alternative method for treating local infections. MGH has antimicrobial, antioxidant, and anti-inflammatory properties and is thus ideal for the treatment of infected wounds $[14,39,40]$.

Alleviation of unpleasant symptoms that accompany non-healing wounds is important to improve the quality of a patient's life [5]. Pain has not only a sensory component, but also an emotional component, which is associated with anxiety, depression, aggression, feelings of danger, helplessness, hopelessness, and loss of motivation [41,42]. Patients with wounds can also suffer from procedural pain [43]. Stress experienced during wound management increases cortisol levels, and this has a negative effect on wound healing [44,45]. In all patients, the pain was alleviated or eliminated after the first dressing change and the intensity of procedural pain was reduced. In all patients, the need for analgesic treatment was gradually reduced or eliminated. As supported by others, this can be attributed to the MGH that prevents incorporation of the wound dressing into the wound bed and subsequent damage of the new granulation during wound dressing [14,21].

Odour is another unpleasant symptom of infection in non-healing wounds. Wound odour has a negative effect on the patient's psyche, is usually associated with abundant production of exudate, and the two factors can lead to social isolation of the patient [21]. In our study, a reduced odour and exudate was noticeable after the first dressing change (the shortest dressing interval was two days and odour and exudate disappeared after approximately 16 days of treatment). Only in patient number 5 was maceration of the wound edges $(0.5 \mathrm{~cm}$ from the wound edge) observed. We believe that the patient applied a large amount of L-Mesitran ${ }^{\circledR}$ Ointment to the wound and did not refresh the dressings frequently enough, which led to the accumulation of exudate in the secondary bandage and, subsequently, maceration. Therefore, it is always important to check exudate levels regularly and change dressings accordingly. After training, this issue was partially resolved, but there was low compliance in this patient. The high sugar content of MGH attracts lymph fluid and wound exudate out of the tissue and helps in the removal of exudate into the dressing [21]. This process, together with the anti-inflammatory activity of MGH, subsequently reduces oedema and pain [21]. Wound odour is produced by bacteria that metabolize serum, tissue proteins, and dead cells, leading to amino acid production and unpleasant odour [46,47]. Glucose in MGH acts as an alternative odourless substrate for these bacteria and thus eliminates odour [21,32,46,47]. In addition, the antimicrobial activity of honey will reduce the number of bacteria in the wound, thereby reducing odour $[46,48]$. This property is most evident within $24 \mathrm{~h}$ after the application of honey to the wound [49]. Also, in our study, patients reported a reduction in odour already after the first wound dressing (the shortest dressing interval was two days). Due to the beneficial healing, it was possible to extend the intervals between the individual dressings to up 
to four days. This of particular importance during the COVID-19 pandemic, when the availability of health services and personal contact with patients is really limited and often replaced by online or phone consultations. In order to get the best result, it is essential that the patients and their relatives cooperate with the treatment regimen and the hygiene measurements. Keeping them involved, maintaining regular appointments, and seeing progression in different aspects (odour, pain, wound progression) helps to keep the patients motivated. Non-healing wounds are an economic burden on healthcare systems. Extending the time between wound dressings, shortening the wound healing time, not administering antibiotics, and providing the ability to perform wound dressings at home (natural social environment or with the help of home care nurses) can significantly reduce the costs. MGH should be considered when non-healing wounds stay stagnant.

\section{Materials and Methods \\ Patient Selection}

In a prospective observational study, MGH was applied to a selected group of patients with non-healing wounds. Inclusion criteria were having a non-healing wound with treatment lasting more than 6 weeks, type 2 diabetes mellitus, presence of local signs of inflammation, absence of systemic sings inflammation, pain including procedural pain, and patient agreement. Exclusion criteria were having an allergy to bee stings or MGH, systemic signs of inflammation, and patient disagreement.

A total of nine patients were included in the study, of which six were men and three women, the average age was 57 years (minimum 43 and maximum 75, with a median of 54 years). Six patients had leg ulcers, two had diabetic foot syndrome, and one had a dehisced surgical wound. Previous therapy consisted of iodine material in six cases, bioceramic dressing in one patient, and nano-silver material in two patients. All patients showed local signs of wound infection and signs of systemic infection were absent. The average assessment of pain intensity during the day reached 5.1 points and procedural pain averaged 7.1 points according to VAS. Most patients $(n=8)$ received oral analgesic treatment during the initial phase of wound treatment until pain levels were minimaldetailed descriptions about the pain level are part of the case summaries. In one patient, the pain was not pharmacologically treated, diabetic neuropathy was present.

Wound swabs were taken using the Levine technique upon first presentation to determine bacterial colonization [50]. Standard laboratory protocols and molecular testing using matrix-assisted laser desorption/ionization time of flight mass spectrometry (MALDIToF MS) were followed to identify the microorganisms and to determine their susceptibility and resistance profiles [51].

Wound characteristics and relevant patient information from the presented nine cases are summarized in Supplementary Table S1. Basic information about the patient, local assessment of the non-healing wound, pain, and the presence and cause of infection are available. Since all wounds were non-healing and locally infected, topical treatment with L-Mesitran was recommended.

\section{Conclusions}

In our prospective case series, we confirmed in a group of nine patients on an outpatient basis that MGH treatment has beneficial effects on the healing process of infected non-healing wounds of various aetiologies. The application of an MGH-containing dressing led to the activation of the healing process, stimulation of debridement, and a faster cleansing phase of the wound bed. MGH reduced odour and exudate secretion and maintained an optimal moist wound bed environment. Wound-related pain and procedural pain were significantly reduced and analgesia was reduced or stopped in all patients. Despite the local signs of infection and the presence of different microorganisms, MGH was effective to resolve infection, and thus replaced the need for antibiotics. Topical treatment of non-healing wounds with MGH dressing led to a lower frequency of wound dressings at home and lower financial costs of care. The healing and reduction in symptoms strongly 
improved the patients' quality of life. MGH forms an attractive alternative to antibiotics to fight infections while enhancing the wound healing trajectory.

Supplementary Materials: The following are available online at https:/ /www.mdpi.com/article/10 .3390/antibiotics10080918/s1. Table S1: Overview of the presented cases.

Author Contributions: Conceptualization, A.H. and A.P.; methodology, A.H. and A.P.; validation, L.C. (Lucie Chlupáčová) and L.C. (Lada Cetlová); investigation, A.H. and A.P.; data curation, A.H., L.C. (Lucie Chlupáčová), and A.P.; writing—original draft preparation A.H.; writing—review and editing, L.C. (Lucie Chlupáčová), L.C. (Lada Cetlová), N.A.J.C. and A.P.; supervision, A.P.; project administration, A.H. and A.P.; final approval of the manuscript, all authors. All authors have read and agreed to the published version of the manuscript.

Funding: This research received no external funding.

Institutional Review Board Statement: The study was conducted according to the guidelines of the Declaration of Helsinki. Anonymized data were collected from study participants and processed in accordance with Act No. 101/2000 Coll. of valid legislation of the Czech Republic. The study was approved by the local ethics committee (the approval does not include the IRB number from the ethics committee, which is common practice in the healthcare facility involved).

Informed Consent Statement: The patients were informed about the study, and they all gave written consent to use their photos and data for publication, providing their anonymity was guaranteed. The principles of the World Medical Association's Declaration of Helsinki were followed.

Data Availability Statement: The data that support the findings of this study are available from the corresponding author upon reasonable request. All data relevant to the study are included in the article. The data are safely stored as requested by Czech legislation in healthcare provider secured electronic system.

Acknowledgments: Authors would like to thank to all the patients involved in the study.

Conflicts of Interest: N.A.J.C. is employed by Triticum Exploitatie BV, the manufacturer of the MGH-based product used in this study. However, he was not involved in the design of the study; the collection, analysis, or interpretation of the data; and the presentation of the results. He was solely consulted for his experience and expertise with MGH. All other authors declare no conflict of interest.

\section{References}

1. World Union of Wound Healing Societies (WUWHS). Florence Congress, Position Document. Advance in Wound Care: The Triangle of Wound Assessment. Wounds Int. 2016. Available online: https:/ /www.coloplast.sg/Documents/Wound/WUWHS_ POSITION\%20DOCUMENT.pdf (accessed on 27 July 2020).

2. Pokorna, A.; Benesova, K.; Muzik, J.; Jarkovsky, J.; Dusek, L. Data Sources for Monitoring of Non-healing Wounds in a National Heal Information System-Epidemiology of Non-healing Wounds Analysis of the National Register of Hospitalized Patients in 2007-2015. Cesk. Slov. Neurol. 2017, 80, 8-17. [CrossRef]

3. Deufert, D.; Graml, R. Disease-specific, health-related quality of life (HRQoL) of people with chronic wounds-A descriptive cross-sectional study using the Wound-QoL. Wound Med. 2017, 16, 29-33. [CrossRef]

4. Krupova, L.; Pokorna, A. Quality of life in patient with non-healing wounds, with particular focus on assesment tools-a literature review. Cent. Eur. J. Nurs. Midwifery 2020, 11, 94-103. [CrossRef]

5. Woo, K.Y.; Krasner, D.L.; Kennedy, B.; Wardle, D.; Moir, O. Palliative wound care management strategies for palliative patients and their circles of care. Adv. Skiin Wound Care 2015, 28, 130-140, quiz 140-132. [CrossRef] [PubMed]

6. Gray, L. Standardising accurate wound assessment and documentation. J. Community Nurs. 2020, 34, 14.

7. McGuckin, K.R.; IMI National Guidelines A Guide to Good Practice Wound Management Photography. Institute of Medical Illustrators. 2019. Available online: https://www.imi.org.uk/wp-content/uploads/2019/04/2019_Apr_IMINatGuidelines_ Wound-Management.pdf (accessed on 10 May 2021).

8. Anghel, E.L.; Kumar, A.; Bigham, T.E.; Maselli, K.M.; Steinberg, J.S.; Evans, K.K.; Kim, P.J.; Attinger, C.E. The Reliability of a Novel Mobile 3-dimensional Wound Measurement Device. Wounds 2016, 28, 379-386. [PubMed]

9. Pokorna, A.; Leaper, D. Assessment and documentation of non-healing, chronic wounds in inpatient health care facilities in the Czech Republic: An evaluation study. Int. Wound J. 2015, 12, 224-231. [CrossRef]

10. Schreml, S.; Szeimies, R.M.; Prantl, L.; Karrer, S.; Landthaler, M.; Babilas, P. Oxygen in acute and chronic wound healing. Br. J. Derm. 2010, 163, 257-268. [CrossRef]

11. Guo, S.; Dipietro, L.A. Factors affecting wound healing. J. Dent. Res. 2010, 89, 219-229. [CrossRef] 
12. Dowsett, C.; Hallern, B. The Triangle of Wound Assessment: A holistic framework from wound assessment to management goals and treatments. Wounds Int. 2017, 8, 34-39.

13. Cremers, N.; Belas, A.; Santos Costa, S.; Couto, I.; de Rooster, H.; Pomba, C. In vitro antimicrobial efficacy of two medical grade honey formulations against common high-risk meticillin-resistant staphylococci and Pseudomonas spp. pathogens. Vet. Derm. 2020, 31, 90-96. [CrossRef]

14. Smaropoulos, E.; Cremers, N.A.J. Treating severe wounds in pediatrics with medical grade honey: A case series. Clin. Case Rep. 2020, 8, 469-476. [CrossRef]

15. Bianchi, T.; Wolcott, R.D.; Peghetti, A.; Leaper, D.; Cutting, K.; Polignano, R.; Rosa Rita, Z.; Moscatelli, A.; Greco, A.; Romanelli, M.; et al. Recommendations for the management of biofilm: A consensus document. J. Wound Care 2016, 25, 305-317. [CrossRef] [PubMed]

16. Sibbald, R.G.; Williamson, D.; Orsted, H.L.; Campbell, K.; Keast, D.; Krasner, D.; Sibbald, D. Preparing the wound beddebridement, bacterial balance, and moisture balance. Ostomy Wound Manag. 2000, 46, 14-22.

17. Strohal, R.; Dissemond, J.; Jordan O’Brien, J.; Piaggesi, A.; Rimdeika, R.; Young, T.; Apelqvist, J. EWMA document: Debridement. An updated overview and clarification of the principle role of debridement. J. Wound Care 2013, 22, 5. [CrossRef]

18. Lumbers, M. Wound debridement: Choices and practice. Br. J. Nurs. 2018, 27, S16-S20. [CrossRef] [PubMed]

19. Stryja, J. The Importance and Limits of the Pressure Ulcer Surgical Debridement. Česká A Slov. Neurol. A Neurochir. 2016, 79, S25-S27. [CrossRef]

20. Manna, B.; Nahirniak, P.; Morrison, C.A. Wound Debridement. In StatPearls [Online]; StatPearls Publishing: Treasure Island, FL, USA, 2021. Available online: https: / /www.ncbi.nlm.nih.gov/books/NBK507882/ (accessed on 10 June 2021).

21. Nair, H.K.R.; Tatavilis, N.; Pospisilova, I.; Kucerova, J.; Cremers, N.A.J. Medical-Grade Honey Kills Antibiotic-Resistant Bacteria and Prevents Amputation in Diabetics with Infected Ulcers: A Prospective Case Series. Antibiotics 2020, 9, 529. [CrossRef]

22. Hermanns, R.; Mateescu, C.; Thrasyvoulou, A.; Tananaki, C.; Wagener, F.A.D.T.G.; Cremers, N.A.J. Defining the standards for medical grade honey. J. Apic. Res. 2020, 59, 125-135. [CrossRef]

23. Nolan, V.C.; Harrison, J.; Cox, J.A.G. Dissecting the Antimicrobial Composition of Honey. Antibiotics 2019, 8, 251. [CrossRef]

24. Gethin, G.; Cowman, S. Bacteriological changes in sloughy venous leg ulcers treated with manuka honey or hydrogel: An RCT. J. Wound Care 2008, 17. [CrossRef] [PubMed]

25. Hermanns, R.; Cremers, N.A.J.; Leeming, J.P.; van der Werf, E.T. Sweet Relief: Determining the Antimicrobial Activity of Medical Grade Honey Against Vaginal Isolates of Candida albicans. J. Fungi 2019, 5, 85. [CrossRef]

26. Oryan, A.; Alemzadeh, E.; Moshiri, A. Biological properties and therapeutic activities of honey in wound healing: A narrative review and meta-analysis. J. Tissue Viability 2016, 25, 98-118. [CrossRef] [PubMed]

27. Yilmaz, A.C.; Aygin, D. Honey Dressing in Wound Treatment: A Systematic Review. Complement. Med. 2020, 51, 102388. [CrossRef]

28. Olsson, M.; Jarbrink, K.; Divakar, U.; Bajpai, R.; Upton, Z.; Schmidtchen, A.; Car, J. The humanistic and economic burden of chronic wounds: A systematic review. Wound Repair. Regen. 2019, 27, 114-125. [CrossRef]

29. Kelechi, T.; Prentice, M.; Madisetti, M.; Brunette, G.; Mueller, M. Palliative Care in the Management of Pain, Odor, and Exudate in Chronic Wounds at the End of Life: A Cohort Study. J. Hosp. Palliat. Nurs. 2017, 19, 17-25. [CrossRef]

30. Lu, J.; Turnbull, L.; Burke, C.M.; Liu, M.; Carter, D.A.; Schlothauer, R.C.; Whitchurch, C.B.; Harry, E.J. Manuka-type honeys can eradicate biofilms produced by Staphylococcus aureus strains with different biofilm-forming abilities. PeerJ 2014,2 , e326. [CrossRef]

31. Pleeging, C.C.F.; Coenye, T.; Mossialos, D.; De Rooster, H.; Chrysostomou, D.; Wagener, F.A.D.T.G.; Cremers, N.A.J. Synergistic Antimicrobial Activity of Supplemented Medical-Grade Honey against Pseudomonas aeruginosa Biofilm Formation and Eradication. Antibiotics 2020, 9, 866. [CrossRef] [PubMed]

32. Biglari, B.; Moghaddam, A.; Santos, K.; Blaser, G.; Buchler, A.; Jansen, G.; Langler, A.; Graf, N.; Weiler, U.; Licht, V.; et al. Multicentre prospective observational study on professional wound care using honey (Medihoney). Int. Wound J. 2013, 10, 252-259. [CrossRef]

33. Halstead, F.D.; Webber, M.A.; Oppenheim, B.A. Use of an engineered honey to eradicate preformed biofilms of important wound pathogens: An in vitro study. J. Wound Care 2017, 26, 442-450. [CrossRef] [PubMed]

34. Wu, Y.K.; Cheng, N.C.; Cheng, C.M. Biofilms in Chronic Wounds: Pathogenesis and Diagnosis. Trends Biotechnol. 2019, 37, 505-517. [CrossRef] [PubMed]

35. Burmolle, M.; Thomsen, T.R.; Fazli, M.; Dige, I.; Christensen, L.; Homoe, P.; Tvede, M.; Nyvad, B.; Tolker-Nielsen, T.; Givskov, M.; et al. Biofilms in chronic infections-A matter of opportunity-Monospecies biofilms in multispecies infections. FEMS Immunol. Med. Microbiol. 2010, 59, 324-336. [CrossRef]

36. Smaropoulos, E.; Cremers, N.A.J. The pro-healing effects of medical grade honey supported by a pediatric case series. Complement. Med. 2019, 45, 14-18. [CrossRef]

37. Combarros-Fuertes, P.; Fresno, J.M.; Estevinho, M.M.; Sousa-Pimenta, M.; Tornadijo, M.E.; Estevinho, L.M. Honey: Another Alternative in the Fight against Antibiotic-Resistant Bacteria? Antibiotics 2020, 9, 774. [CrossRef] [PubMed]

38. de Groot, T.; Janssen, T.; Faro, D.; Cremers, N.A.J.; Chowdhary, A.; Meis, J.F. Antifungal Activity of a Medical-Grade Honey Formulation against Candida auris. J. Fungi 2021, 7, 50. [CrossRef] 
39. Smaropoulos, E.; Cremers, N.A.J. Medical grade honey for the treatment of extravasation-induced injuries in preterm neonates-A case series. Adv. Neonatal. Care 2020, in press.

40. Lu, J.; Cokcetin, N.N.; Burke, C.M.; Turnbull, L.; Liu, M.; Carter, D.A.; Whitchurch, C.B.; Harry, E.J. Honey can inhibit and eliminate biofilms produced by Pseudomonas aeruginosa. Sci. Rep. 2019, 9, 18160. [CrossRef]

41. Sochor, M.; Slama, O. Management of chronic and acute pain in patients with cancer diseases. Klin. Onkol. 2015, 28, 94-98. [CrossRef] [PubMed]

42. Juric Vukelic, D.; Juric, J. Hydrocolloid Dressing Application in the Treatment of Chronic Wounds and Relation to Quality of Life. Acta Clin. Croat. 2017, 56, 544-549. [CrossRef] [PubMed]

43. Rosenbaum, C. An overview of integrative care options for patients with chronic wounds. Ostomy Wound Manag. 2012, 58, 44-51.

44. Edwards, J. Dealing with wound-related pain at dressing change. J. Community Nurs. 2013, 27, 36-42.

45. Upton, D.; Solowiej, K.; Hender, C.; Woodyatt, K.Y. Stress and pain associated with dressing change in patients with chronic wounds. J. Wound Care 2012, 21, 53-54, 56,58 passim. [CrossRef] [PubMed]

46. Alam, F.; Islam, M.A.; Gan, S.H.; Khalil, M.I. Honey: A potential therapeutic agent for managing diabetic wounds. Evid. Based Complement Altern. Med. 2014, 2014, 169130. [CrossRef] [PubMed]

47. Yapucu Gunes, U.; Eser, I. Effectiveness of a honey dressing for healing pressure ulcers. J. Wound Ostomy Cont. Nurs. 2007, 34, 184-190. [CrossRef]

48. Molan, P.C. Re-introducing honey in the management of wounds and ulcers-theory and practice. Ostomy Wound Manag. 2002, $48,28-40$.

49. Labban, L. Honey as a promising treatment for diabetic foot ulcers (DFU). J. Med. Soc. 2014, 28, 64-68. [CrossRef]

50. Angel, D.E.; Lloyd, P.; Carville, K.; Santamaria, N. The clinical efficacy of two semi-quantitative wound-swabbing techniques in identifying the causative organism(s) in infected cutaneous wounds. Int. Wound J. 2011, 8, 176-185. [CrossRef] [PubMed]

51. Singhal, N.; Kumar, M.; Kanaujia, P.K.; Virdi, J.S. MALDI-TOF mass spectrometry: An emerging technology for microbial identification and diagnosis. Front. Microbiol. 2015, 6, 791. [CrossRef] 